\title{
Design of a Scalable Integrated Nanophotonic Electron Accelerator on a Chip
}

\author{
Uwe Niedermayer $\odot,{ }^{1},{ }^{*}$ Jan Lautenschläger $\odot,{ }^{1}$ Thilo Egenolf $\odot,{ }^{2}$ and Oliver Boine-Frankenheim ${ }^{1,2}$ \\ ${ }^{1}$ Institut für Teilchenbeschleunigung und Elektromagnetische Felder, Technische Universität Darmstadt, \\ Schlossgartenstrasse 8, 64289 Darmstadt, Germany \\ ${ }^{2}$ GSI Helmholtzzentrum für Schwerionenforschung GmbH, Planckstrasse 1, 64291 Darmstadt, Germany
}

(Received 9 June 2021; accepted 21 July 2021; published 12 August 2021)

\begin{abstract}
A simple way of implementing a scalable laser-driven nanophotonic electron accelerator on a chip is presented. The design requires only a single incident laser pulse and can be fabricated straightforwardly on commercial silicon-on-insulator wafers. We investigate the low-energy regime of tabletop electron microscopes where the silicon structures safely allow peak gradients of about $150 \mathrm{MeV} / \mathrm{m}$. By means of a three-dimensional alternating-phase-focusing scheme, we obtain about half of the peak gradient as the average gradient with six-dimensional confinement and full-length scalability. The structures are completely designed within the device layer of the wafer and can be arranged in stages. We choose the stages as energy doublers and outline how errors in the handshake between the stages can be corrected by on-chip steerers. Since the electron pulse length in the attosecond realm is preserved, our chip is the ideal energy booster for ultrafast-electron-diffraction machines, opening the megaelectronvolt scale on tabletop setups.
\end{abstract}

DOI: 10.1103/PhysRevApplied.16.024022

\section{INTRODUCTION}

Dielectric laser accelerators (DLAs) were proposed as long ago as 1962 [1,2]. However, experimental demonstrations of the high acceleration gradients due to short pulses and high material damage thresholds came 50 years later [3,4] by means of modern femtosecond lasers and lithographic nanofabrication. Recent advances have further pushed the peak gradients in nanophotonic structures for relativistic electrons to $690 \mathrm{MeV} / \mathrm{m} \mathrm{[5]} \mathrm{and} 850 \mathrm{MeV} / \mathrm{m}$ [6] and to $133 \mathrm{MeV} / \mathrm{m}$ [7] and $370 \mathrm{MeV} / \mathrm{m}$ [8] for their subrelativistic counterparts. While DLAs in high-energy accelerator facilities are an emerging subject of study $[9,10]$, there are already many imaginable applications of subrelativistic DLAs in electron microscopy and diffraction [11-13]. Especially an electron diffractometer [14,15] could be equipped with a DLA to allow working at significantly higher energy, while the entire setup, including the laser, would still be tabletop-sized. Higher energy in electron diffraction translates to higher spatial resolution [16] and is nowadays restricted to extremely large electrostatic setups or bulky rf systems with issues of their own, e.g., jitter [17].

*niedermayer@temf.tu-darmstadt.de

Published by the American Physical Society under the terms of the Creative Commons Attribution 4.0 International license. Further distribution of this work must maintain attribution to the author(s) and the published article's title, journal citation, and DOI.
To enable scalable DLAs for these use cases, the setup has to be made simple and robust in operation. The amount of external equipment has to be reduced as much as possible. For example, on-chip waveguides [18,19], which might increase the efficiency of an already-working accelerator, are also avoided here for robustness reasons. In the long run, we imagine a setup and a timeline oriented on the history of the integrated circuit, which first needed much external equipment but nowadays is a stand-alone device working with nearly $100 \%$ reliability. Pursuing this goal, we deem robustness and scalability as more critical than efficiency or the number of features and thus construct the accelerator according to the beam dynamics necessities to minimize beam losses for given injector parameters.

The accelerating near fields in a DLA decay exponentially from the grating surface, and thus such accelerators have only tiny (subwavelength) apertures. Keeping the electrons in this aperture cannot be achieved by magnets, since tremendous focusing gradients on the order of several million teslas per meter would be required [20-22]. A solution to this problem is to use the evanescent fields from the nanophotonic structures themselves and arrange them such that they keep the electron beam in the channel without external focusing being required. This is achieved by the alternating-phase-focusing (APF) technique [21,23], which presents a design recipe for the chip as a composition of individual cells, to which periodic boundary conditions can be applied in a good approximation. 
To use most of the small physical aperture (i.e., to maximize the dynamic aperture), the three-dimensional- (3D) APF scheme [23] requires the synchronous electric field to be symmetric in both transverse planes. When driven with two lasers from both lateral sides, this results in a rather strict requirement for the two lasers to have exactly the same phase, as otherwise the working point is laterally dislocated from the channel center. Moreover, dual lateral illumination requires a significant amount of experimental complexity, especially when an independently driven on-chip buncher is used [24,25].

In this paper we present how the symmetric fields required for a scalable APF DLA can be produced in a simple manner, entirely encoded in the chip. The chip itself can be fabricated by a standard process of nanophotonics - namely, etching of solely the top layer of commercially available silicon-on-insulator (SOI) wafers by electron-beam lithography and/or photolithography. By shining the laser from the top, the partial reflection from the silicon substrate is used to create a symmetric quadrupolar synchronous field to enable a stable $3 \mathrm{D}$ lattice design [23] on an asymmetrically (single pulse) illuminated chip.

Additionally to the structure and accelerator lattice design, we present computationally heavy full 3D start-toend simulations of the setup, exposing the imperfections of the structure, which eventually lead to emittance increase and beam losses. For these heavy simulations we use CST Particle Studio [26] and we compare the results with those of lightweight simulations in DLAtrack6D [27].

We find that due to the imperfect vertical symmetry, a deflection force is exerted, which can be partially compensated by the structure design. The remaining vertical deflection is sufficiently small in a single-stage setup. For a multistage setup, it can be compensated by on-chip steerers located between the stages. Multiple stages can be constructed on a single SOI chip, enabling full scalability. Because of the lithographic fabrication process, the lateral and longitudinal alignment is perfect. Vertical misalignment can be corrected by electrostatic deflectors, which can be fabricated as silicon "bridges" on a silicon-on-glass wafer that is mounted from the top. The required steering voltages can be connected by pads on the device layer of the SOI wafer far from the laser spot.

This paper is organized as follows: Sec. II presents the top-illuminated SOI structures that are crucial for the entire setup and explains how they are combined into a scalable accelerator using the 3D-APF segmentation technique introduced in Ref. [23]. This procedure is performed and a full start-to-end simulation of the resulting energy doubler for electron microscopes is shown in Sec. III. In Sec. IV, we show how the issue of a coherent deflection force, which leads to partial beam loss, can be overcome. Section V outlines a full integration of the setup, including staging of multiple energy doublers and beam steering between the stages. Finally, Sec. VI concludes with an outlook of controlling the remaining few external parameters by a digital-twin model and enabling ultrafast electron diffraction on the megaelectronvolt level with tabletop setups based on the chips introduced here.

\section{TOP-ILLUMINATED 3D-APF STRUCTURES ON SOI WAFERS}

The principle of the setup is shown in Fig. 1. A laser pulse of wavelength $\lambda=2 \mu \mathrm{m}$ impinges as a plane wave from the top (i.e., in the negative $x$ direction), with the polarization in the $z$ direction. The structures are quasiperiodic, with period lengths $\lambda_{g}=\beta \lambda$, where $\beta=v / c$ is the synchronous electron velocity. The cells are arranged in APF-type segments [21], acting as accelerators and thick focusing lenses. As in many other photonic devices produced on SOI wafers, it is crucial here that the oxide layer (brown in Fig. 1) has a refractive index $n_{\mathrm{SiO}_{2}}=1.45$ [28] that differs as much as possible from that in the silicon device layer and substrate $\left(n_{\mathrm{Si}}=3.41\right.$ [28]). The former is important for the structures to stand alone and produce a vertically symmetric field as if the pillars were free-floating, and the latter is necessary for a high reflection factor. Throughout this work we use a commercially available SOI wafer made of a $700-\mu \mathrm{m}$ silicon substrate, a 3- $\mu \mathrm{m}$ buried oxide layer, and a $220-\mathrm{nm}$ silicon device layer [29]. At higher energy, thicker device layers and wider channels would also be feasible, allowing larger emittances and higher particle throughput.
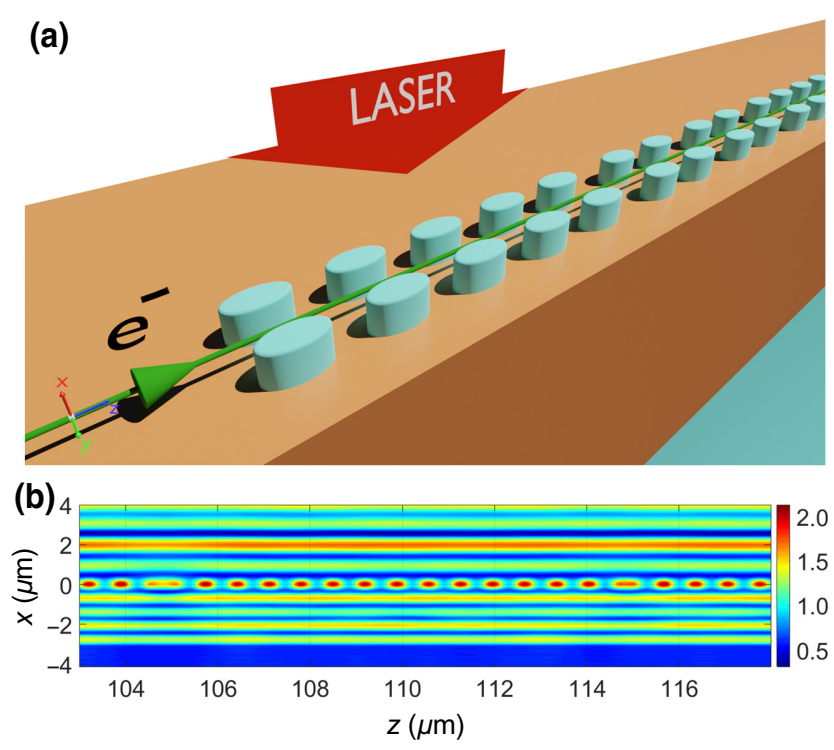

FIG. 1. Acceleration structures created by etching only the device layer of a SOI wafer (a) and longitudinal electric field magnitude normalized to the incident laser field strength in a longitudinal cut $(y=0$ plane) (b). 

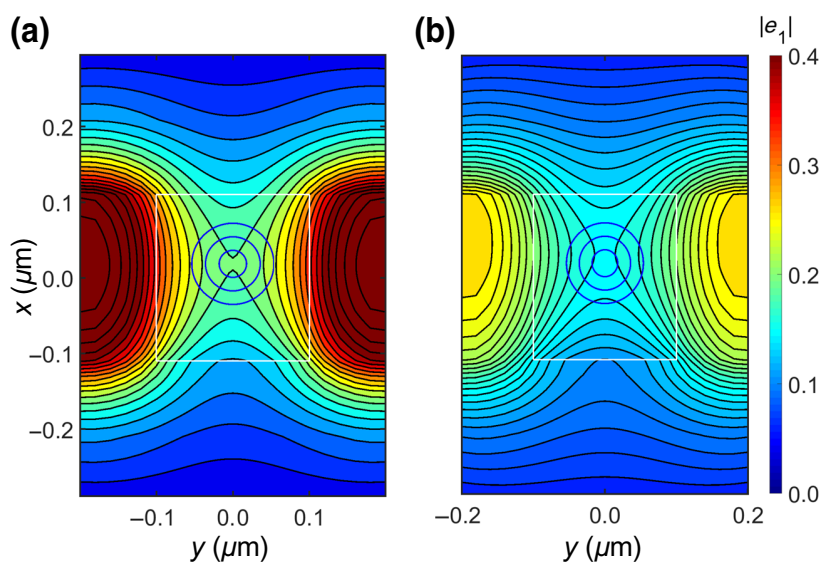

FIG. 2. Normalized synchronous longitudinal electric field distribution for $\beta=0.31$ (a) and uncorrected $\beta=0.5$ (b). The white box indicates the beam channel (note the refraction of the potential lines at the bottom). The blue circles indicate $1 \sigma, 2 \sigma$, and $3 \sigma$ of the beam size at injection.

The structure cells are characterized by the first spatial harmonic of the longitudinal electric field

$$
e_{1}(x, y)=\frac{1}{\lambda_{g}} \int_{-\lambda_{g} / 2}^{\lambda_{g} / 2} E_{z}(x, y, z) e^{2 \pi i z / \lambda_{g}} d z
$$

where $E_{z}(x, y, z)$ is the electric field in the frequency domain, which we however determine from a time-domain simulation and on-the-fly Fourier transform. This is particularly efficient, since the speed of explicit time-domain simulation is exploited, while not saving the entire time dependence of the three-dimensional field. The higher (nonsynchronous) spatial harmonics can be ignored [21]. By our determining $e_{1}$ in each cell and fixing the synchronous phase $\phi_{s}$ as $\pm 60^{\circ}$ off-crest, the acceleration ramp and the 3D focusing channel are designed [23] such that half the peak gradient is available as the average gradient within one segment.

The structures shown in Fig. 1 locally create a quadrupolar distribution (see Fig. 2) as $e_{1}(x, y)=$ $e_{10} \cosh \left(k_{y} y\right) \cos \left(k_{x} x\right)$, which refers to the "counterphase" APF scheme. Since we deal only with this scheme throughout this paper, we take $k_{x}$ and $k_{y}$ as purely real. From Ref. [23], the identification $k_{x}=\operatorname{Re} k_{x}$ and $k_{y}=\operatorname{Im} k_{y}$ has to be made, yielding $k_{x}^{2}-k_{y}^{2}+\omega^{2} /(\beta \gamma c)^{2}=0$, with $\omega=2 \pi c / \lambda$ being the laser angular frequency.

\section{DESIGN OF AN ENERGY DOUBLER FOR ELECTRON MICROSCOPES}

For the design of a scalable accelerator, the fields as shown in Fig. 1 (bottom) can be calculated for each individual cell of length $\lambda_{g}$, where periodic boundary conditions are used in the longitudinal direction and open boundary conditions are used in the $x$ direction, in which the $z$-polarized laser beam travels. The wave impedance of the open boundary condition at the bottom is matched to that of silicon. Because the wafer substrate is much thicker than the laser pulse length, reflections at the very bottom (which also has high surface roughness) can be neglected. Laterally, at $y=0$ and $y=6 \mu \mathrm{m}$, magnetic boundary (symmetry) conditions are used. The magnitude of the synchronous harmonic is plotted in Fig. 2 for two different beam velocities. As desired, the structure cells produce a quadrupolelike field between the pillars. To enable the design procedure as outlined in Ref. [23], the vertical drift of the working point $x_{0}(\beta)$ needs to be compensated. Insufficient compensation of this drift results in a coherent oscillation of the beam, leading to emittance increase and beam loss.

The periods are elliptic cylinders of the device-layer height $h=220 \mathrm{~nm}$ and radii $r_{y}=100 \mathrm{~nm}$ and variable $r_{z}$. By changing $r_{z}=r_{z 0}+\Delta r_{z}$, one can change the working-point position exactly such that the drift with $\beta$ is compensated:

$$
\Delta x_{0}=\frac{\partial x_{0}}{\partial r_{z}} \Delta r_{z}+\frac{\partial x_{0}}{\partial \beta} \Delta \beta=0 .
$$

Since the drift of the working point is not too large and continuously depends on $\beta$ and $r_{z}$, we can linearize this numerically determined dependency and obtain $r_{z}=r_{z 0}-$ $\xi\left(\beta-\beta_{0}\right)$, with $\xi=\partial_{\beta} x_{0} / \partial_{r_{z}} x_{0}=-1.7 \mu \mathrm{m}$ at a linearization point $\beta_{0}=0.42$ and $r_{z 0}=0.35 \mu \mathrm{m}$. A comparison of the corrected and uncorrected single-cell parameters is shown in Fig. 3. Moreover, this plot also contains a comparison between sharp top edges and realistically rounded top edges. As one can see, the effect in $e_{1}$ is small and the shift in $x_{0}$ is constant (i.e., correctable).

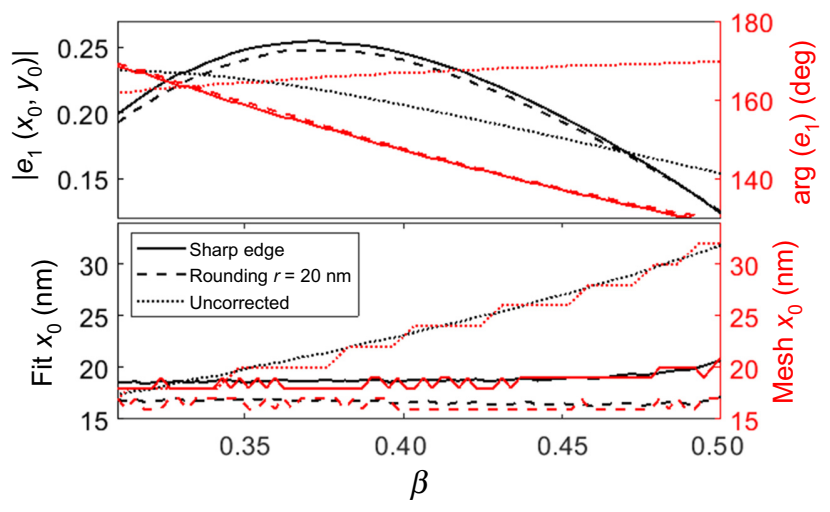

FIG. 3. Synchronous electric field and position of the working point $x_{0}$ versus $\beta$. The solid lines show the corrected [Eq. (2)] cells, as compared with the uncorrected ones (dotted lines). The dashed line shows the effect of applying a realistic rounding (20 $\mathrm{nm}$ ) to the top edge of the elliptical cylinders. The working-point position $x_{0}$ is determined in two well-agreeing ways: taking the numerical maximum of the data (mesh $x_{0}$ ) and fitting a parabola to the data (fit $x_{0}$ ). 
We design an accelerator with injection energy $W_{\text {inj }}=$ $26.478 \mathrm{keV}\left(\beta_{\text {inj }}=0.31\right)$, which doubles the energy to 53 $\mathrm{keV}$, or $\beta=0.4233$. Continuation to triple energy ( $\beta=$ 0.5 ) is possible straightforwardly; however, simulation of this entire setup currently exceeds our computational capabilities. The designed stage has a length of $381 \mu \mathrm{m}$ and an average gradient of $69.3 \mathrm{MeV} / \mathrm{m}$ at a design incident laser field strength $E_{L}$ of $600 \mathrm{MV} / \mathrm{m}$. To stay below the damage threshold of silicon (the damage threshold for $\mathrm{SiO}_{2}$ is significantly higher), the laser pulse needs to be in the subpicosecond realm. This can be achieved by pulse-front tilting (PFT) [30-32], where the phase fronts and the polarization remain parallel to the wafer. However, since the electron velocity increases, the PFT angle $\alpha=\arctan (1 / \beta)$ has to decrease accordingly. Since our structure has a finite length, the required curved pulse front can be approximated by one linear PFT per stage, which however requires a finite (minimum) pulse length [23]. We find the optimum $\alpha=69.8^{\circ}$ and a pulse length of $430 \mathrm{fs}$, which keeps the local laser amplitude above $95 \%$ of the maximum; see Fig. 4. The pulse width is chosen here to be $\sigma_{z}=6 \mathrm{~mm}$, and $\sigma_{y}$ should be larger than several tens of micrometers for practical alignment purposes. The fluence of this pulse is about $200 \mathrm{~J} / \mathrm{m}^{2}$, which is almost an order of magnitude below the damage threshold of silicon $[33,34]$.

To match the needs of cylindrical symmetric electronmicroscope setups, the beam spot at the input is designed

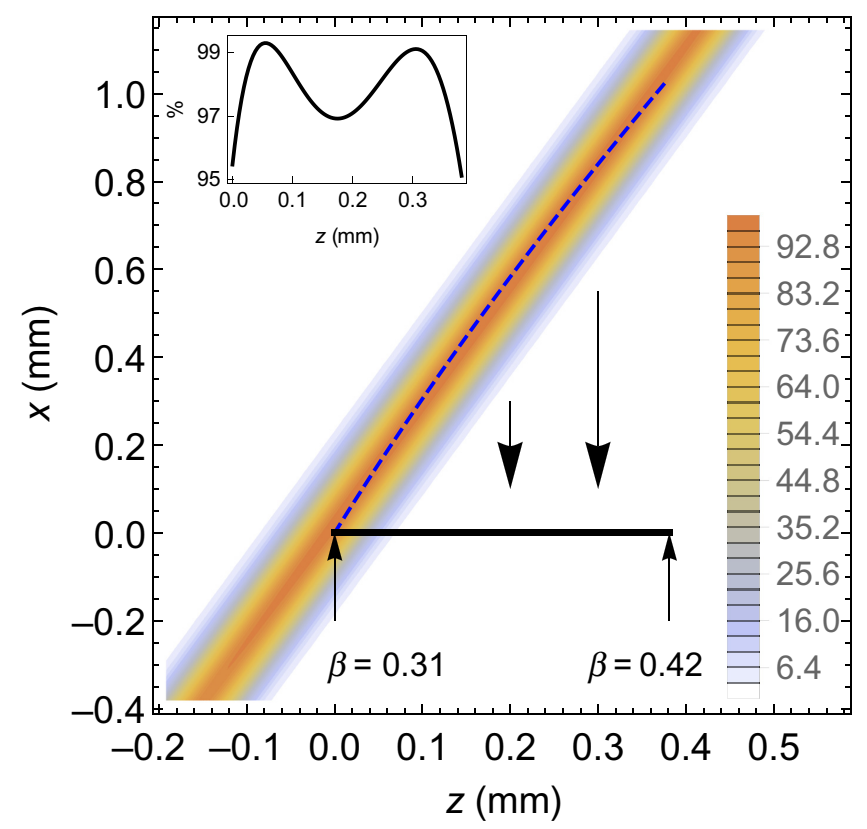

FIG. 4. Electric field amplitude as a percentage of the maximum for the tilted laser pulse. The accelerator structure is indicated by the thick black line and the phase fronts are parallel to it. The dashed blue line describes the position in the laser pulse that is synchronous with an electron in the accelerator. The inset shows the laser amplitude on this curve. to be circular $\left(\hat{\beta}_{x}=\hat{\beta}_{y}=32 \mu \mathrm{m}\right)$. Moreover, by use of half segments, the beam can be made uncorrelated at the input $(\hat{\alpha}=0)$ and output $(\hat{\alpha} \approx 0)$. From a DLAtrack6D [27] simplified simulation, we expect a Gaussian beam with geometric emittances $\varepsilon_{x}=\varepsilon_{y}=10 \mathrm{pm}$ and bunch length $\sigma_{z}=10 \mathrm{~nm}$ (matched energy and angle spreads) to have an energy-doubling throughput of about $50 \%$. In modern electron microscopes such low emittances are available [35] and the bunching can be achieved by on-chip bunchers [25]. By further lowering of the emittances, the throughput of the accelerator structures can be increased toward $100 \%$. Animations of the DLAtrack6D simulations are available in Supplemental Material [36].

Before running a complete 3D tracking simulation of the entire setup, we first calculate the fields in the entire setup (approximately $350 \times 10^{6}$ hexahedral mesh cells) and retrieve the channel position and the focusing wave numbers $k_{x}$ and $k_{y}$ as well as $e_{1}$ from them. As seen in Fig. 5, the agreement with the calculation for the individual cells with use of periodic boundary conditions is excellent. At the APF phase jumps, an unavoidable Gibbs phenomenon is however visible, which among other effects might degrade the throughput.

The designed envelopes as well as the tracking results are shown in Figs. 6 and 7. In the bottom plots, the acceleration ramp and a loss comparison of the tracking in the full fields and in DLAtrack6D are shown. The envelopes relate to the lattice functions as $a_{x, y, \Delta z}=$ $\left(\hat{\beta}_{x, y, z} \varepsilon_{x, y, z}\right)^{1 / 2}$ and $a_{x^{\prime}, y^{\prime}, \Delta z^{\prime}}=\left(\hat{\gamma}_{x, y, z} \varepsilon_{x, y, z}\right)^{1 / 2}$, where the

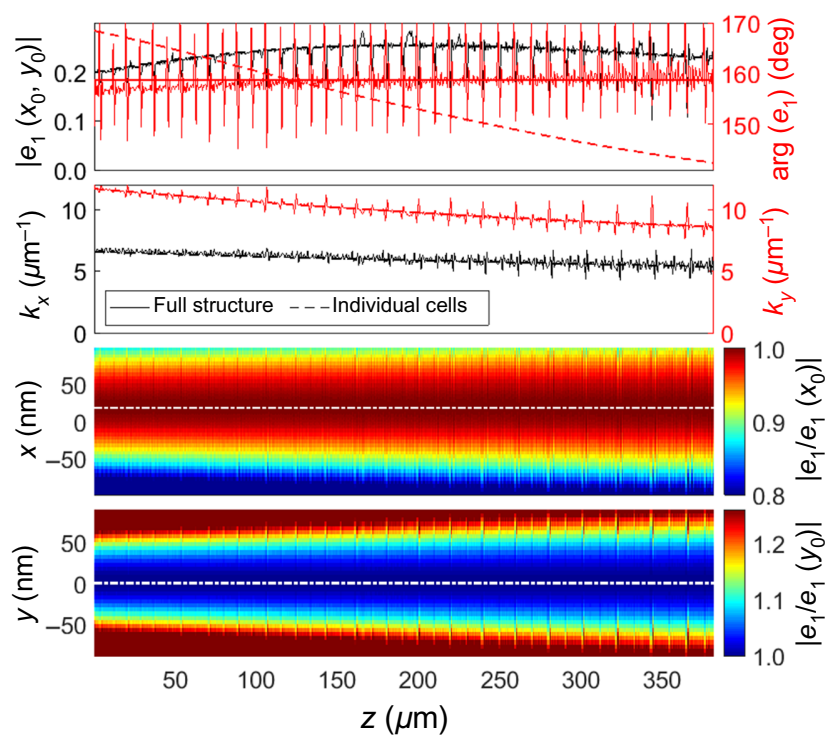

FIG. 5. Comparison of the field quality for single cells with periodic boundaries and the whole structure. The phase drift is corrected by our displacing the pillars within the cell [23] and thus follows a straight line. The two bottom plots show the channel position, which is almost perfectly straight at $x_{0}=18 \mathrm{~nm}$ and $y_{0}=0$. 


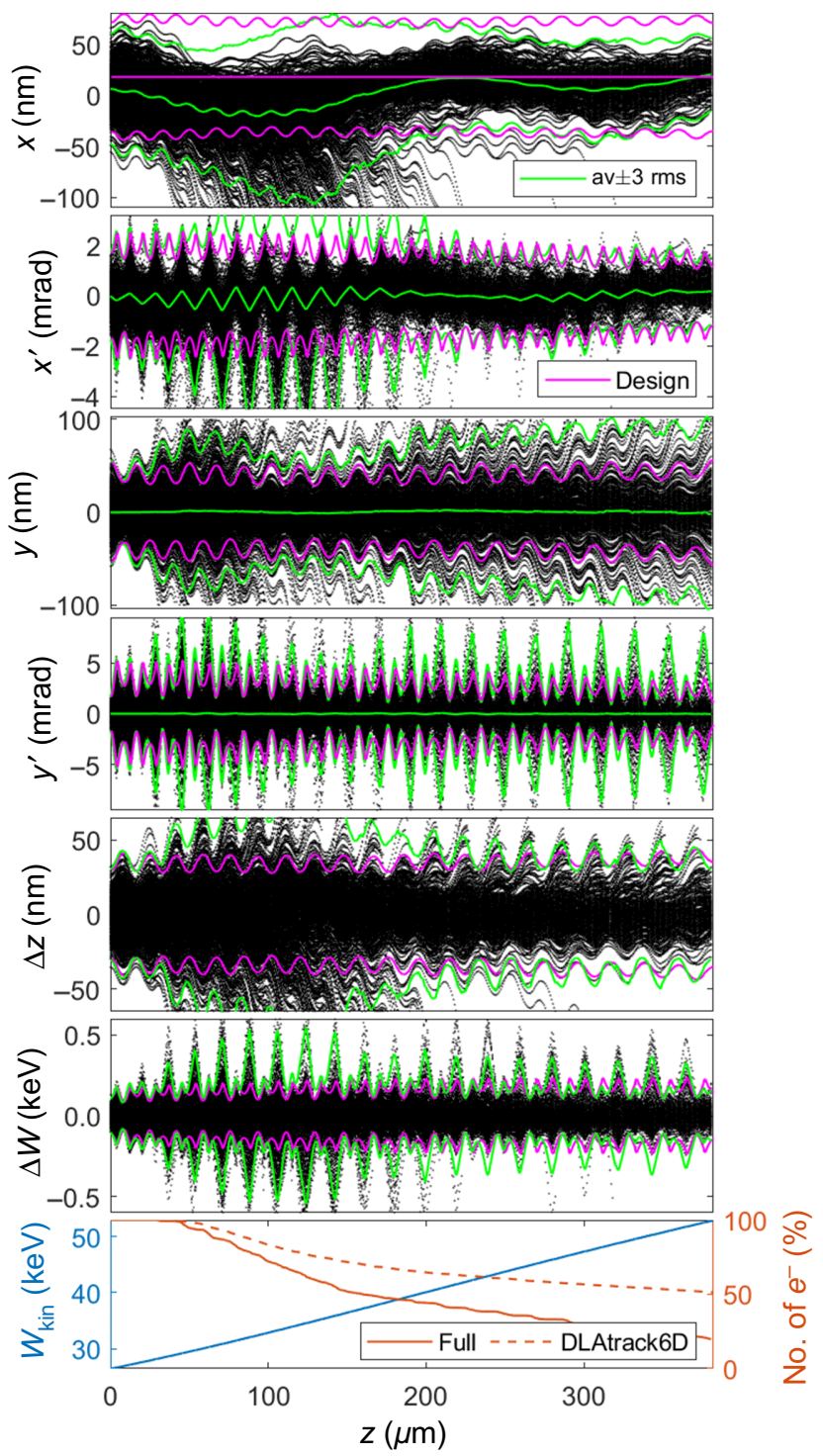

FIG. 6. Phase-space evolution in the elliptical-cell structure without a correction field. The black dots show individual particle positions and the lines show the averages and the averages with 3 times the rms value added or subtracted. Magenta is for the design, whereas green is for the statistics obtained from the black-dot data. The slight off-axis injection increases the throughput to $19.8 \%$, as compared with the very poor transmission for on-axis injection.

geometric emittances undergo adiabatic damping as $\varepsilon=$ $\varepsilon_{0} \beta_{0} \gamma_{0} / \beta \gamma$ and the longitudinal emittance is obtained from the initial bunch length as $\varepsilon_{z}=\sigma_{z}^{2} / \hat{\beta}_{z}$. The matched energy spread reads $\Delta W=m c^{2} \beta^{2} \gamma^{3} a_{\Delta z^{\prime}}$. The same initial six-dimensional bunch distributions are used in the fast DLAtrack6D simulation and in the full-field tracking simulation, where the bunch is released at $z=0$ and appropriate time to hit the synchronous phase. In both simulations the particles are treated independently; we choose 1000 (for unbunched 10000 ) electrons to obtain good statistics. In

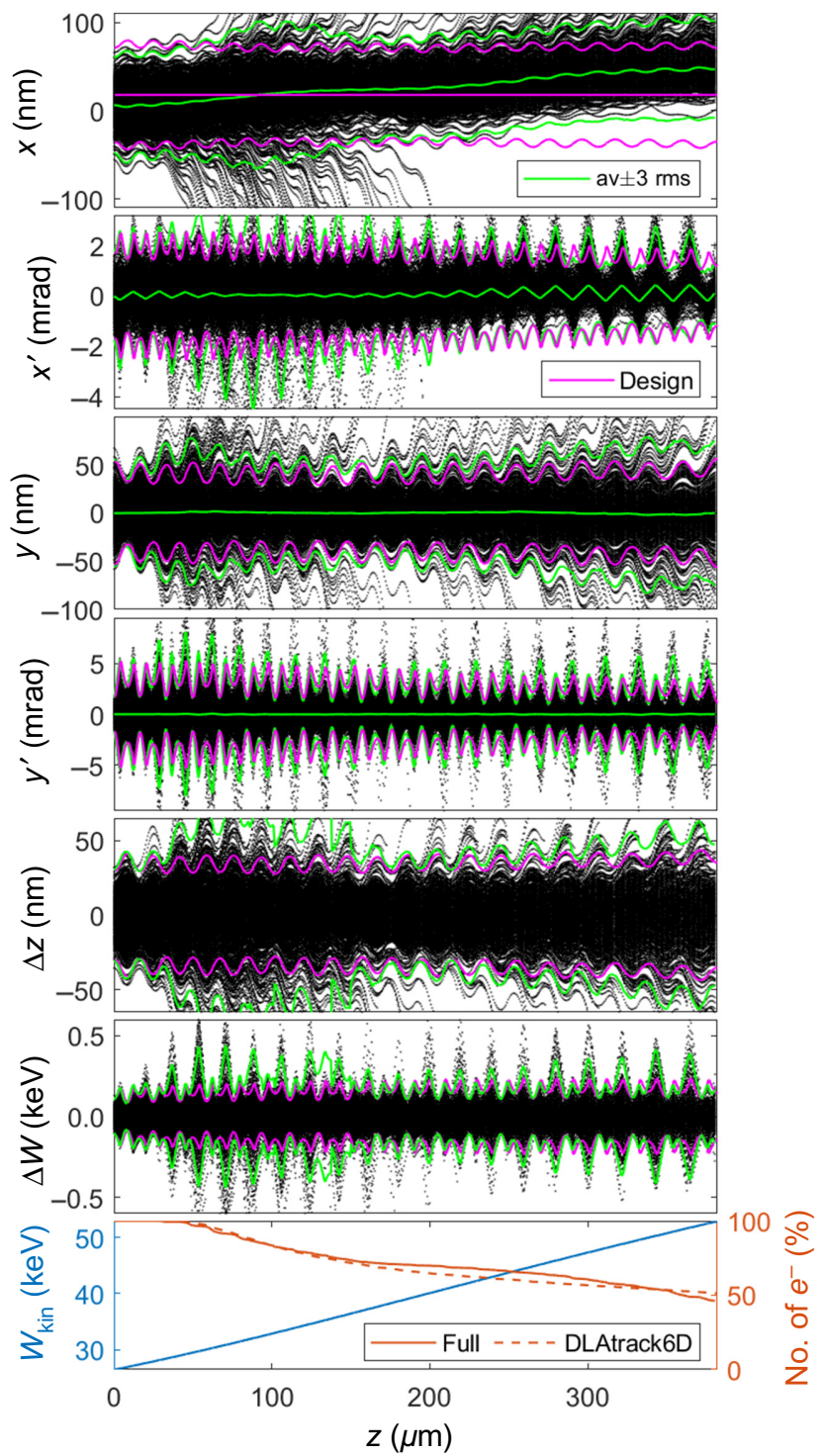

FIG. 7. Phase-space evolution in the elliptical-cell structure with $14 \mathrm{mT}$ correction field. The black dots show individual particle positions and the lines show the averages and the averages with 3 times the rms value added or subtracted. Magenta is for the design, whereas green is for the statistics obtained from the black-dot data. The throughput can be increased to $46.1 \%$, almost as high as anticipated by the fast DLAtrack6D simulation. For small particle amplitudes, the DLAtrack6D simulation produces the same envelopes as the design [21,23] (not shown here).

reality there is only one electron per bucket at most, however there are many buckets per pulse and a high pulse repetition rate. This renders collective effects entirely irrelevant for subrelativistic DLAs, unlike in relativistic DLAs, where collective effects were recently investigated by an extension of DLAtrack6D [37].

As seen in the top panel in Fig. 6, the electron beam experiences a strong deflection force in the negative $x$ direction. After a length of $100 \mu \mathrm{m}$ and some beam loss, 
the focusing is however able to catch the beam and move it back to the designed trajectory. Coherent motion due to mismatch in the $x$ direction might also lead to emittance increase and beam loss in the $y$ or $z$ direction, since the aperture-filling high particle amplitudes probe the coupled nonlinearities of the fields [27].

The origin of the deflecting force is the gradient of the phase across $x$, i.e., $\nabla \arg e_{1}(x, y)$, leading to a deflection ramp similar to the acceleration ramp. Following Eq. (12) in Ref. [27], the transverse acceleration can be written as

$$
x^{\prime \prime}=-\left.\frac{q E_{L} \lambda_{g}}{m \gamma \beta^{2} c^{2}}\left|e_{1}\right| \cos \phi_{s} \partial_{x} \arg \left(e_{1}\right)\right|_{x_{0}},
$$

where $\left.\partial_{x} \arg \left(e_{1}\right)\right|_{x_{0}}$ is the linearized phase gradient on the reference beam axis. The resulting effect is similar to that of a homogeneous magnetic field, which leads to a deflection $x^{\prime \prime}=q B_{y} / m \gamma \beta c$. Therefore, with $T=\lambda / c$, the magnetic field required to correct for the deflection reads

$$
B_{\text {corr }}=\left.E_{L} T\left|e_{1}\right| \cos \phi_{s} \partial_{x} \arg \left(e_{1}\right)\right|_{x_{0}},
$$

which is only implicitly dependent on the beam velocity via $\left.\left|e_{1}\right| \partial_{x} \arg \left(e_{1}\right)\right|_{x_{0}}$. Figure 8 shows the required correction field as a function of $\beta$. The inset shows the worst case for injection at $\beta=0.31$, where the phase change across the beam size is roughly $2^{\circ}$, resulting in a required correction field of more than $100 \mathrm{mT}$.

A correcting external magnetic field cannot however be applied to each cell as would be required; it can be applied only homogeneously over the entire chip. Therefore, the correction needs to be a compromise between

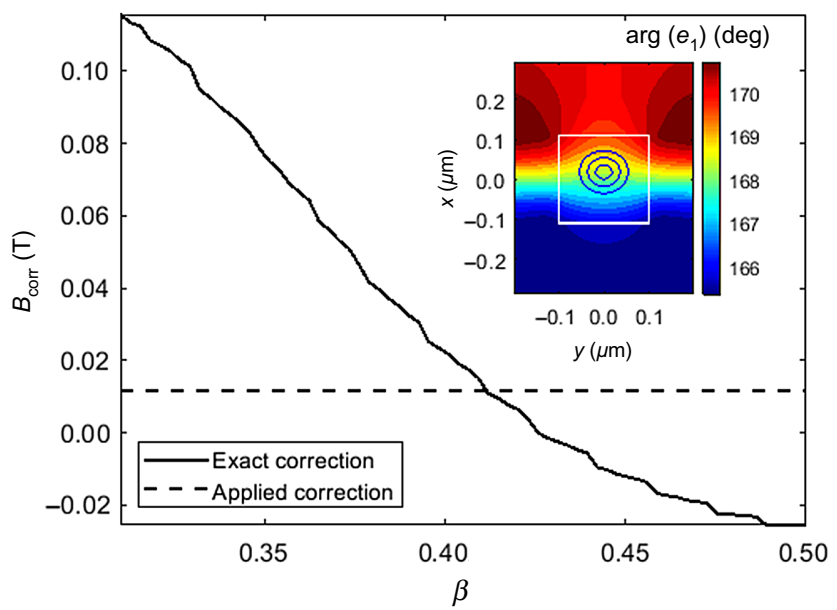

FIG. 8. Required correction field due to the phase gradient. The inset shows the phase distribution across the channel at the injection energy $(\beta=0.31)$. Since the correction field can be applied only in a homogeneous manner, a compromise has to be found, which is $B=14 \mathrm{mT}$, and a slightly displaced injection uses the focusing channel for further correction. the different values along the channel. Additionally, an offaxis injection can be used to counteract the initially strong deflection. An example of doing this is shown in Fig. 7, where a 14-mT field is applied and the injection is chosen at $x_{0, \text { inj }}=6 \mathrm{~nm}, 12 \mathrm{~nm}$ below the design value. With this, the throughput is more than doubled from $19.8 \%$ to $46.1 \%$, which is however still slightly lower than the prediction by DLAtrack6D of $50.8 \%$ disregarding the deflection.

\section{COMPROMISE BETWEEN STRAIGHT CHANNEL AND LOW COHERENT DEFLECTION}

As seen in Fig. 8, the coherent deflection and the required correction field are strongest at the lowest beam velocity, where also most of the channel correction is applied. However, modification of the ellipse-semiaxis radii does not lead to a sufficient reduction. Especially at low energy, the phase gradient can rather be reduced by reshaping the pillars as rectangles $(\Delta Y=440 \mathrm{~nm}$ by $\Delta Z=460 \mathrm{~nm}$ and $R=40 \mathrm{~nm}$ corner rounding for fabrication feasibility), reducing the phase gradient to about a tenth of the value for the elliptical pillars at injection, which is plotted in Fig. 9. In the plot we also investigate lateral grounding traces to remove lost electrons from the pillars. The lateral grounding traces even further reduce the
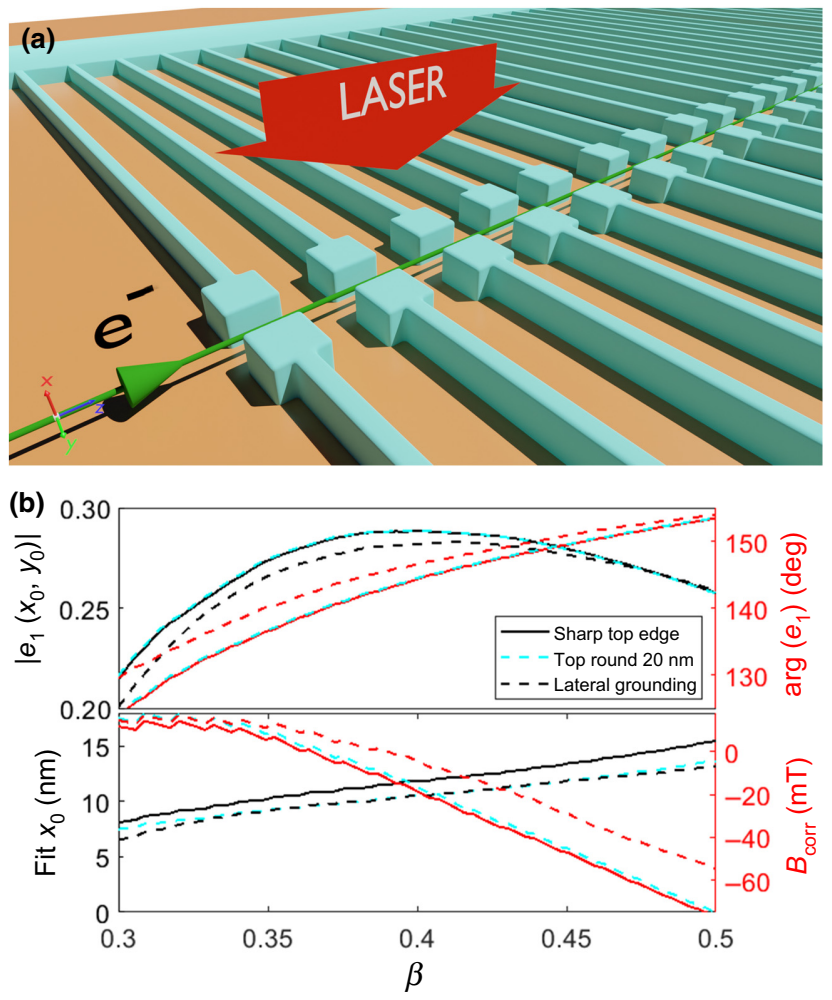

FIG. 9. Rectangular acceleration structures with lateral grounding (a) and velocity scan (b). The sharp-top-edge structures without grounding are compared with ones with lateral grounding and with top rounding. 
phase gradient-induced deflection. Moreover, in Fig. 9 we once again verify that the effect of a realistic top rounding of $20 \mathrm{~nm}$ is small. In the full simulation that follows, we keep nongrounded structures with sharp top edges for simplicity.

The lattice is redesigned in the same way as for the elliptical structures. The working-point drift for the rectangular structures is only $5 \mathrm{~nm}$ over the entire length of the energy-doubler structure. Correction of this slight drift would lead to an increase in deflection force and is therefore not attempted. In the field-flatness analysis shown in Fig. 10 this drift is hardly visible. Because of the higher $e_{1}$, the length is reduced to $344 \mu \mathrm{m}$ (average gradient 77.7 $\mathrm{MeV} / \mathrm{m}$ ) and the throughput predicted by DLAtrack6D is $57.3 \%$.

The full simulation as shown in Fig. 11 results in a throughput of $44.6 \%$, without any correction magnetic field. Further optimization can be done by slightly changing the injection angle $x_{0}^{\prime}$, injection position $x_{0}$, and injection phase. Moreover, another tuning parameter is the laser amplitude, which gives the highest throughput at about 3\% above the nominal value (i.e., $618 \mathrm{MV} / \mathrm{m}$ ). Increasing the laser amplitude does not lead to higher output energy since the ramp is hard-coded in the lattice. However, it leads to a steeper potential well and thus to a smaller but hotter beam. Since this however appears nonuniformly, it leads to a "sweet spot" being close to the design incident field.

In the simulations here, we assume a bunched beam, which can be readily created by APF buncher structures [25]. These bunchers can also be created with 3D confinement on the same SOI chip. Depending on how "adiabatic" the buncher is designed to be (i.e., how long it is in the end), a bunching efficacy of $50 \%$ can be achieved

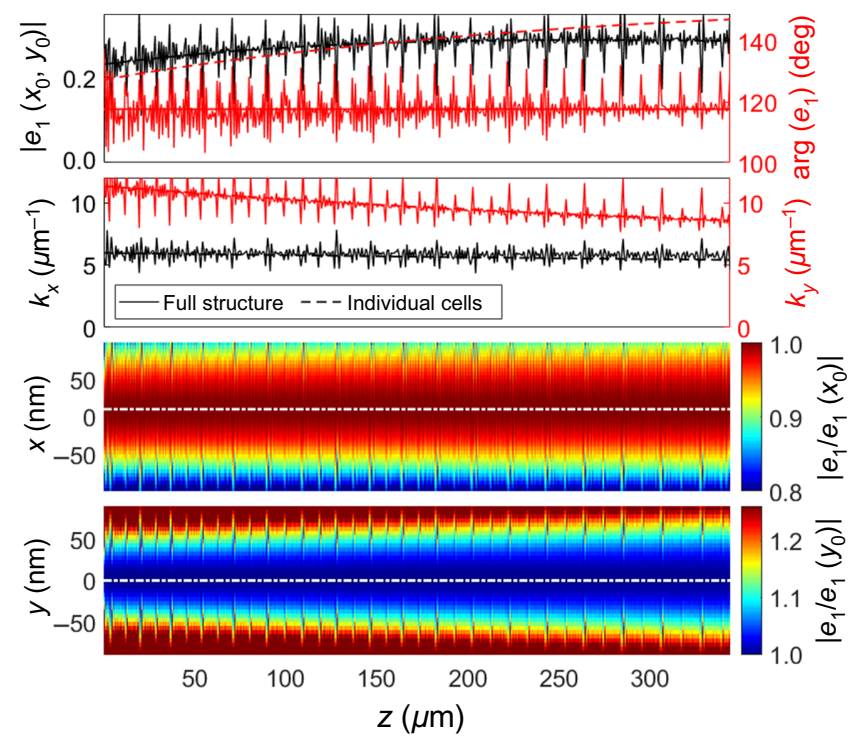

FIG. 10. Field-flatness analysis as in Fig. 5 for the rectangularcell structure. The 5-nm drift of $x_{0}$ is too small to be visible.

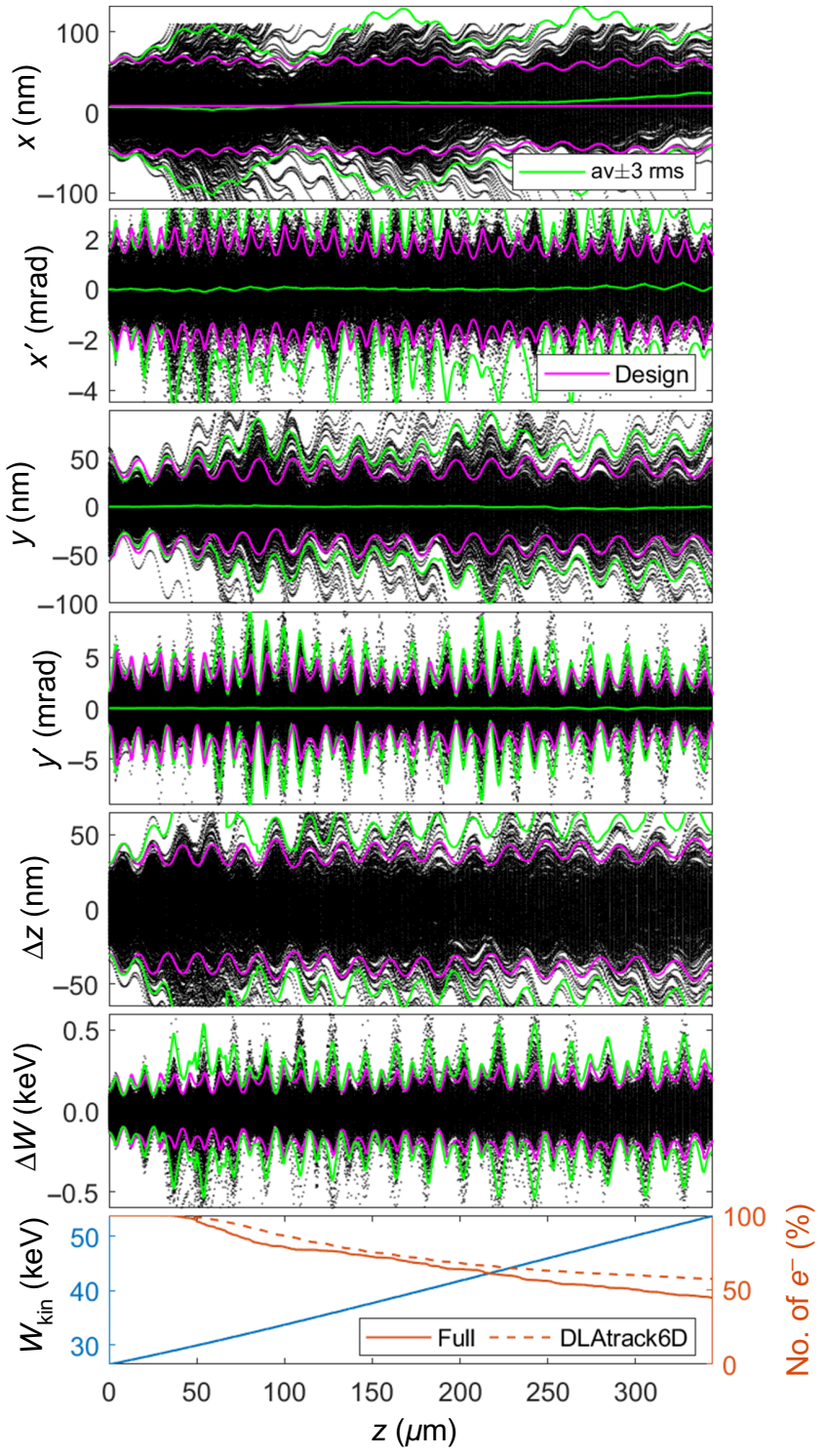

FIG. 11. Evolution of the electron beam in the design with rounded rectangular structures. The expected transmission can almost be reached without any correcting external field.

with bunch lengths of several hundreds of attoseconds and matched energy spreads. For an unbunched beam, DLAtrack6D predicts transmission of $5.1 \%$, and the full simulation results in $4.0 \%$. Thus, one would expect a combined transmission of about $20 \%$ with a buncher as compared with $4 \%$ without a buncher for the 10-pm emittance beam. Video files of the DLAtrack6D and CST Particle Studio simulations are available in Supplementary Material [36].

\section{VERTICAL STEERERS AND STAGING THE DESIGN ON A SINGLE CHIP}

We have seen that structures can be made that achieve decent energy-doubling throughput without any correcting field for available beam emittances of $10 \mathrm{pm}$ at $\beta=0.31$. 
To obtain higher energy, the setup can be staged, where each stage can be run with its own constant PFT angle. At the output of each stage, the beam might however be vertically displaced and inclined. For injection into the next stage, the vertical injection angle and position need to be controlled. For that purpose we exploit the fact that the substrate is grounded and thus constitutes an electrode of an electrostatic deflector. The device layer can act as a connection platform for the other electrode. Moreover, the device layer can also be equipped with lateral traces so as to ground the acceleration structures (see Fig. 9). This allows us to remove lost charges. Lateral grounding is more preferable than connecting the pillars longitudinally, since longitudinal connections decrease the bandwidth, therefore strongly increasing the field deviations at the phase jumps [23].

A mock-up layout for the device layer with the connections is shown in Fig. 12. Both the acceleration structures and the large areas with the crosses are connected to pads located far from the laser spots. They can be contacted by metallic wires or needle contacts. The crosses are alignment marks to align a second chip from the top. The

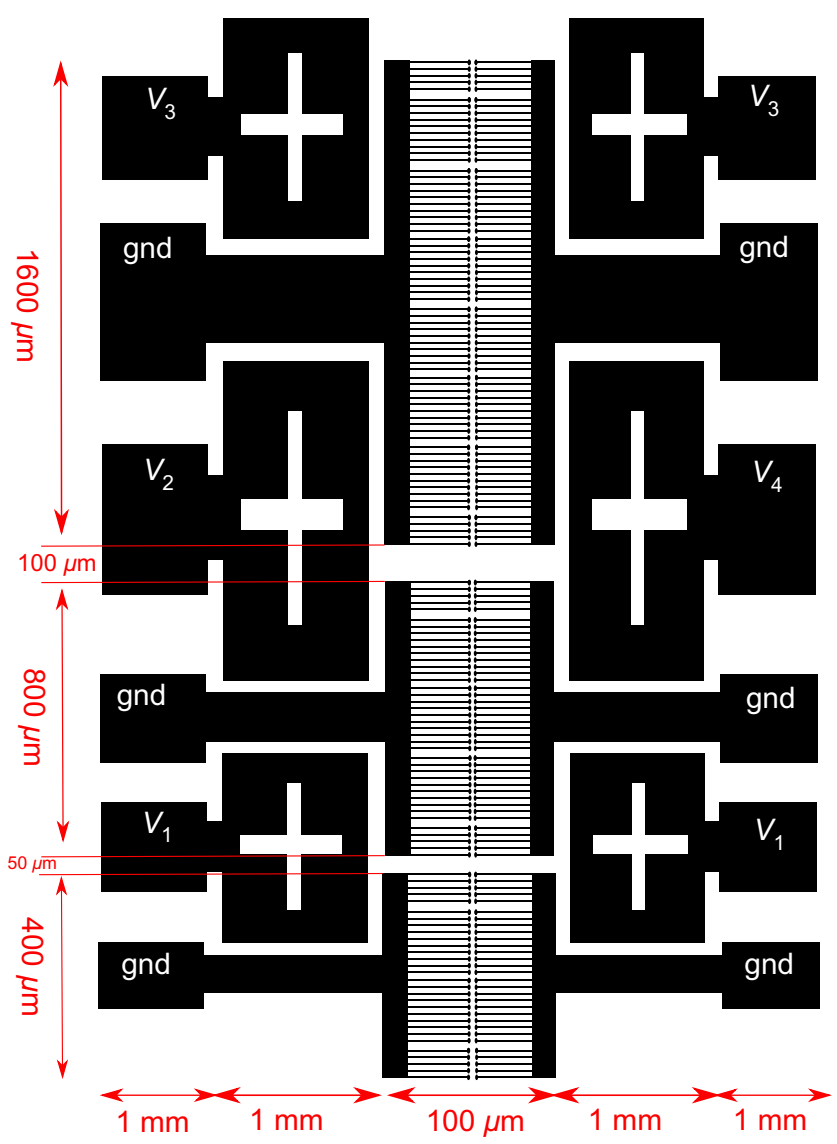

FIG. 12. Layout of the device layer for a staged accelerator chip. The dimensions are mock-up parameters for a three-stage setup with four steering voltages. The drawing is not to scale.

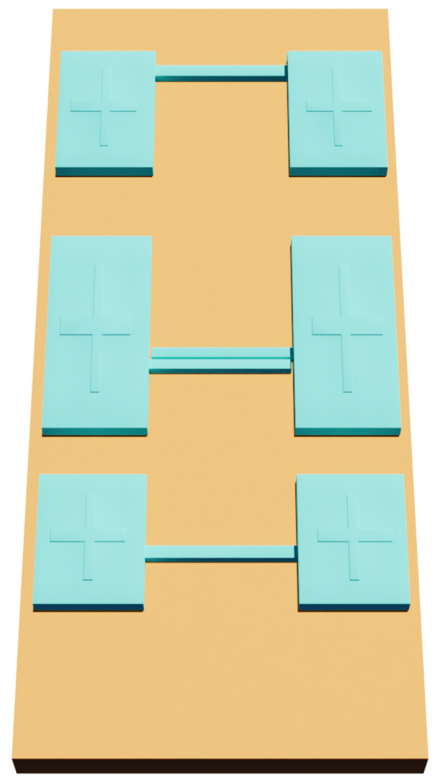

FIG. 13. Deflection electrodes etched on a silicon-on-glass wafer. The large areas with the crosses are supposed to be aligned with and touching the SOI wafer's device layer.

upper chip as shown in Fig. 13 is made on a thicker siliconon-glass wafer, where all the silicon is removed except for some bridges, which constitute the second electrode of the deflectors.

The deflection strength is calculated from the dc breakdown strength of glass, which is $10 \mathrm{MV} / \mathrm{m}$ [38], as $x^{\prime \prime}=$ $q E_{x} / m \gamma \beta^{2} c^{2}=E_{x} /(W / q) / \beta^{2}$. For $53 \mathrm{keV}$, this results in $50 \mathrm{~m}^{-1}$, leading to a kick of $\Delta x^{\prime}=x^{\prime \prime} L=2.5 \mathrm{mrad}$ and a displacement of $\Delta x=x^{\prime \prime} L^{2} / 2=62.5 \mathrm{~nm}$ in a deflector of length $L=50 \mu \mathrm{m}$. The required voltage on the pads is only $30 \mathrm{~V}$ when a $3-\mu \mathrm{m}$ gap is maintained for for the beam, and half of the breakdown field strength is used as deflection field. For fused silica the dielectric strength is about $500 \mathrm{MV} / \mathrm{m}$ [38], which would reduce the required length significantly. In the design of the deflector lengths, other quantities also play a role. For example, the beam defocuses during that drift (i.e., its rms size will increase by $\sigma_{x^{\prime}} L \approx 20 \mathrm{~nm}$ ), which has to be matched to the next stage. Moreover, the two laser spots for the two stages should either or the structures should be designed for constructive interference of the two pulses in the interface region of the two respective stages.

Two deflectors can also be combined to create a dogleg. This can be used to send the beam above the acceleration structures to bypass a subsequent stage. In this manner, the stages can be subsequently tested and aligned from the lowest energy to the highest energy, where all stages are on the same chip. Since longitudinal and lateral alignment between the stages is already perfect due to the high accuracy of the lithographic fabrication of the device layer, the 


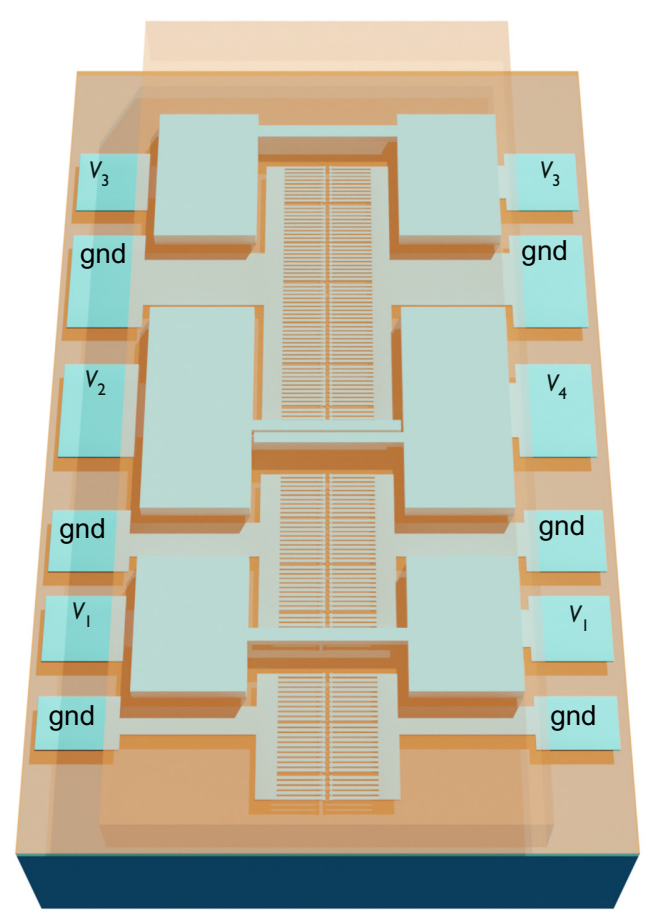

FIG. 14. Final assembly of a three-stage acceleration chip. Each stage is driven by an individually tilted laser pulse from the top.

vertical steerers should suffice to align the beam for a controlled energy boost above $1 \mathrm{MeV}$ in four or five stages with a total length of about $1 \mathrm{~cm}$. The complete assembly of 3 such stages is shown in Fig. 14.

\section{CONCLUSION AND OUTLOOK}

In summary, we show two different designs of nanophotonic linear electron accelerators, which double the energy of tabletop electron microscopes. The chips are less than $400 \mu \mathrm{m}$ in length and contain about 500 DLA cells. The cells are organized by an optimized 3D-APF scheme, which produces smooth and small envelope functions in all directions of the six-dimensional phase space. By means of illuminating the structures from the top and exploiting the partial reflection due to the refractive-index contrast in the SOI wafer, we can drive the energy doubler by a single, linearly tilted laser pulse of 430 -fs length. The experimental complexity is significantly reduced, which also practically enables multistage setups, fabricated on a single chip with perfect longitudinal and lateral alignment. The vertical alignment can be controlled by on-chip electrostatic steerers, which also allow the bypassing of entire stages. The alignment of the top electrodes needs only to be in the single-digit micrometer range longitudinally and in the two-digit micrometer range laterally. The voltage supply of the deflectors as well as grounding of the laserdriven accelerator structures themselves can be achieved by metallic contacting on the SOI device layer far from the laser spot.

We simulate the two single-stage designs both in the simplified, one-kick-per-cell DLA simulator DLAtrack6D [27] and with the full fields in CST Particle Studio [26]. The full simulations reveal that due to the small phase gradient across the beam channel, there is a coherent force deflecting the beam vertically. We find that the impact of this parasitic force is stronger than the effect of the workingpoint drift with changing beam velocity. To compensate for this force, an external homogeneous magnetic field can be applied; however, complete compensation is obtained only for a particular beam velocity. A better approach is to eliminate the phase gradient in the individual cell design. We find that the phase gradient can be significantly reduced by choosing rectangular rather than elliptical structures at low energy. For fabrication reasons the rectangles must have rounded corners though. With these structures, a small channel drift of $5 \mathrm{~nm}$ however remains. Correction of this drift using Eq. (2) leads to a stronger phase gradient, which is not favorable. The excess beam losses found in the full simulations are rather due to the remaining coherent deflection appearing at high energy $(\beta \approx 0.4)$. At this higher energy, the elliptical structures produce zero phase gradient. Thus, to compensate for the phase gradient for the entire ramp, hyperelliptical structures, parameterized by $\left(y / r_{y}\right)^{p}+\left(z / r_{z}\right)^{p}=1$ (with $p=p(\beta)>2$ not necessarily an integer), can be used. Varying $p$ smoothly allows us to design structures that look like rectangles at low energy and smoothly translate into ellipses at higher energy when $p$ approaches 2 .

The full simulations performed in this study are heavy for twofold reasons. First, the stages themselves have an electrical length (length in the unit of the wavelength) equal to the number of DLA cells. Second, the channel is transversely much smaller than the entire setup and the beam is very sensitive to its initial parameters within the channel. Although in experiments the shots can come with a repetition rate above $100 \mathrm{kHz}$, a single shot requires about $17 \mathrm{~h}$ for simulation in the full 3D fields. Thus, scanning the sensitive injection parameters (i.e., $x_{0}, x_{0}^{\prime}$, injection phase, and laser amplitude) and eventually also correction fields is extremely time-consuming. In the future one could apply a surrogate model to optimize these parameters with fewer evaluations of the full 3D model ("Bayesian optimization"), as was recently done for plasma accelerators [39]. The numerous shots in the experiments come however with the drawback of only little diagnostic equipment available so far. Essentially, in the experiment one can see the beam on a spectrometer screen only after the accelerator. The laser distribution over the accelerator structure is unknown to sufficient accuracy, without even considering the phase-space evolution within the structures. The advantages of quick results and the observability of all parameters are combined in 
the fast one-kick-per-cell simulation tool DLAtrack6D. In the future, one could imagine using it as a "digital twin" running in parallel with experiments. Of course, the modeling in DLAtrack6D would have to be refined to match input and output data of the experiment. Especially modeling the phase-gradient kick as introduced here would be crucial. The necessary parameters could be obtained from machine learning [40], especially so-called transfer learning, where the training data are obtained by a cheaper process (e.g., numerous DLAtrack6D runs or experimental data). With such a "digital twin" an experimenter could get live insights into otherwise-hidden parameters (e.g., the position in the chip where beam losses occur). This would especially allow tuning of the injection parameters such that the beam is matched and envelope oscillations are largely avoided so as to obtain maximal charge throughput.

To reduce experimental complexity in the first place, the single-stage energy doublers presented here can be elongated to an energy tripler or even longer, depending on the laser pulse length. If greater energy gains at such low energy are desired, a curved pulse-front-tilt is necessary. The curved pulse can be circumvented by approximating it by linear pieces, resulting in a staged setup, where each stage is driven by an individual pulse of the respective linear tilt angle. Fabricating four or five such stages on a common chip allows the energy to be boosted into the megaelectronvolt realm. At the output, the 3D-APF lattice can be designed such that the beam is uncorrelated and can be magnified by a cylindrical electrostatic lens (or Einzel lens) to a larger spot size and to be parallel to the optical axis at the specimen. In this manner, the way is paved to high-energy electron diffraction on tabletop setups.

We have submitted a patent application for the above structures to the German patent office under DE 102020119875.2, priority date July 28, 2020.

\section{ACKNOWLEDGMENTS}

This work is funded by the Gordon and Betty Moore Foundation under Grant No. GBMF4744 (ACHIP) and the German Federal Ministry of Education and Research (Grant No. 05K19RDE).

[1] A. Lohmann, Electron acceleration by light waves, IBM Tech. Note 5, 169 (1962).

[2] K. Shimoda, Proposal for an electron accelerator using an optical maser, Appl. Opt. 1, 33 (1962).

[3] J. Breuer and P. Hommelhoff, Dielectric laser acceleration of $28 \mathrm{keV}$ electrons with the inverse Smith-Purcell effect, Nucl. Instrum. Methods Phys. Res., Sect. A: Accel. Spectrom. Detect. Assoc. Equip. 740, 114 (2013).

[4] E. A. Peralta, K. Soong, R. J. England, E. R. Colby, Z. Wu, B. Montazeri, C. McGuinness, J. McNeur, K. J. Leedle,
D. Walz, E. B. Sozer, B. Cowan, B. Schwartz, G. Travish, and R. L. Byer, Demonstration of electron acceleration in a laser-driven dielectric microstructure, Nature 503, 91 (2013).

[5] K. P. Wootton, Z. Wu, B. M. Cowan, A. Hanuka, I. V. Makasyuk, E. A. Peralta, K. Soong, R. L. Byer, and R. J. England, Demonstration of acceleration of relativistic electrons at a dielectric microstructure using femtosecond laser pulses, Opt. Lett. 41, 2696 (2016).

[6] D. Cesar, S. Custodio, J. Maxson, P. Musumeci, X. Shen, E. Threlkeld, R. J. England, A. Hanuka, I. V. Makasyuk, E. A. Peralta, K. P. Wootton, and Z. Wu, High-field nonlinear optical response and phase control in a dielectric laser accelerator, Commun. Phys. 1, 46 (2018).

[7] P. Yousefi, J. McNeur, M. Kozák, U. Niedermayer, F. Gannott, O. Lohse, O. Boine-Frankenheim, and P. Hommelhoff, Silicon dual pillar structure with a distributed Bragg reflector for dielectric laser accelerators: Design and fabrication, Nucl. Instrum. Methods Phys. Res., Sect. A: Accel. Spectrom. Detect. Assoc. Equip. 909, 221 (2018).

[8] K. J. Leedle, R. Fabian Pease, R. L. Byer, and J. S. Harris, Laser acceleration and deflection of $963 \mathrm{keV}$ electrons with a silicon dielectric structure, Optica 2, 158 (2015).

[9] B. Hermann, S. Bettoni, T. Egenolf, U. Niedermayer, E. Prat, and R. Ischebeck, Laser-driven modulation of electron beams in a dielectric micro-structure for X-ray free-electron lasers, Sci. Rep. 9, 19773 (2019).

[10] B. Marchetti, et al., SINBAD-ARES - a photo-injector for external injection experiments in novel accelerators at DESY, J. Phys.: Conf. Ser. 1596, 012036 (2020).

[11] B. J. Siwick, J. R. Dwyer, R. E. Jordan, and R. J. Miller, An atomic-level view of melting using femtosecond electron diffraction, Science 302, 1382 (2003).

[12] R. F. Egerton, Outrun radiation damage with electrons? Adv. Struct. Chem. Imaging 1, 5 (2015).

[13] T. Gruene, J. T. Wennmacher, C. Zaubitzer, J. J. Holstein, J. Heidler, A. Fecteau-Lefebvre, S. De Carlo, E. Müller, K. N. Goldie, I. Regeni, T. Li, G. Santiso-Quinones, G. Steinfeld, S. Handschin, E. van Genderen, J. A. van Bokhoven, G. H. Clever, and R. Pantelic, Rapid structure determination of microcrystalline molecular compounds using electron diffraction, Angew. Chem. - Int. Ed. 57, 16313 (2018).

[14] J. Heidler, R. Pantelic, J. T. Wennmacher, C. Zaubitzer, A. Fecteau-Lefebvre, K. N. Goldie, E. Müller, J. J. Holstein, E. van Genderen, S. De Carlo, and T. Gruene, Design guidelines for an electron diffractometer for structural chemistry and structural biology, Acta Crystallogr. Section D: Struct. Biol. 75, 458 (2019).

[15] M. Warren, "Why didn't we think to do this earlier?" Chemists thrilled by speedy atomic structures, Nature 563, 16 (2018).

[16] J. Yang, et al., Diffractive Imaging of Coherent Nuclear Motion in Isolated Molecules, Phys. Rev. Lett. 117, 153002 (2016).

[17] R. K. Li and C. X. Tang, Temporal resolution of MeV ultrafast electron diffraction based on a photocathode RF gun, Nucl. Instrum. Methods Phys. Res., Sect. A: Accel. Spectrom. Detect. Assoc. Equip. 605, 243 (2009).

[18] T. W. Hughes, S. Tan, Z. Zhao, N. V. Sapra, K. J. Leedle, H. Deng, Y. Miao, D. S. Black, O. Solgaard, J. S. 
Harris, J. Vuckovic, R. L. Byer, S. Fan, R. J. England, Y. J. Lee, and M. Qi, On-Chip Laser-Power Delivery System for Dielectric Laser Accelerators, Phys. Rev. Appl. 9, 54017 (2018).

[19] N. V. Sapra, K. Y. Yang, D. Vercruysse, K. J. Leedle, D. S. Black, R. J. England, L. Su, R. Trivedi, Y. Miao, O. Solgaard, R. L. Byer, and J. Vuckovic, On-chip integrated laser-driven particle accelerator, Science 367, 79 (2020).

[20] A. Ody, P. Musumeci, J. Maxson, D. Cesar, R. J. England, and K. P. Wootton, Flat electron beam sources for DLA accelerators, Nucl. Instrum. Methods Phys. Res., Sect. A: Accel. Spectrom. Detect. Assoc. Equip. 865, 75 (2017).

[21] U. Niedermayer, T. Egenolf, O. Boine-Frankenheim, and P. Hommelhoff, Alternating-Phase Focusing for DielectricLaser Acceleration, Phys. Rev. Lett. 121, 214801 (2018).

[22] D. S. Black, K. J. Leedle, Y. Miao, U. Niedermayer, R. L. Byer, and O. Solgaard, Laser-Driven Electron Lensing in Silicon Microstructures, Phys. Rev. Lett. 122, 104801 (2019).

[23] U. Niedermayer, T. Egenolf, and O. Boine-Frankenheim, Threedimensional Alternating-Phase Focusing for Dielectric-Laser Electron Accelerators, Phys. Rev. Lett. 125, 164801 (2020).

[24] D. S. Black, U. Niedermayer, Y. Miao, Z. Zhao, O. Solgaard, R. R. L. Byer, and K. J. K. Leedle, Net Acceleration and Direct Measurement of Attosecond Electron Pulses in a Silicon Dielectric Laser Accelerator, Phys. Rev. Lett. 123, 264802 (2019).

[25] U. Niedermayer, D. S. Black, K. J. Leedle, Y. Miao, R. L. Byer, and O. Solgaard, Low Energy Spread Attosecond Bunching and Coherent Electron Acceleration in Dielectric Nanostructures, Phys. Rev. Appl. 15, L021002 (2021).

[26] www.cst.com CST Studio Suite (2021).

[27] U. Niedermayer, T. Egenolf, and O. Boine-Frankenheim, Beam dynamics analysis of dielectric laser acceleration using a fast $6 \mathrm{D}$ tracking scheme, Phys. Rev. Accel. Beams 20, 111302 (2017).

[28] www.refractiveindex.info (2020).

[29] www.order.universitywafer.com (2020).

[30] D. Cesar, J. Maxson, P. Musumeci, X. Shen, R. J. England, and K. P. Wootton, Optical design for increased interaction length in a high gradient dielectric laser accelerator, Nucl. Instrum. Methods Phys. Res., Sect. A: Accel. Spectrom. Detect. Assoc. Equip. 909, 252 (2018).
[31] M. Kozák, J. McNeur, N. Schönenberger, J. Illmer, A. Li, A. Tafel, P. Yousefi, T. Eckstein, and P. Hommelhoff, Ultrafast scanning electron microscope applied for studying the interaction between free electrons and optical near-fields of periodic nanostructures, J. Appl. Phys. 124, 023104 (2018).

[32] Y. Wei, M. Ibison, G. Xia, J. D. A. Smith, and C. P. Welsch, Dual-grating dielectric accelerators driven by a pulse-fronttilted laser, Appl. Opt. 56, 8201 (2017).

[33] P. Pronko, P. V. Rompay, C. Horvath, X. Liu, T. Juhasz, and G. Mourou, Avalanche ionization and dielectric breakdown in silicon with ultrafast laser pulses, Phys. Rev. B 58, 2387 (1998).

[34] K. Soong, R. L. Byer, E. R. Colby, R. J. England, and E. A. Peralta, Laser damage threshold measurements of optical materials for direct laser accelerators, AIP Conf. Proc. 1507, 511 (2012).

[35] A. Feist, N. Bach, N. Rubiano da Silva, T. Danz, M. Möller, K. E. Priebe, T. Domröse, J. G. Gatzmann, S. Rost, J. Schauss, S. Strauch, R. Bormann, M. Sivis, S. Schäfer, and C. Ropers, Ultrafast transmission electron microscopy using a laser-driven field emitter: Femtosecond resolution with a high coherence electron beam, Ultramicroscopy 176, 63 (2017).

[36] U. Niedermayer, J. Lautenschläger, T. Egenolf, and O. Boine-Frankenheim, See Supplemental Material at http://link.aps.org/supplemental/10.1103/PhysRevApplied. 16.024022 for videos of the DLAtrack6D and CST simulations, without a correction field and without initial bunching (2021).

[37] T. Egenolf, U. Niedermayer, and O. Boine-Frankenheim, Tracking with wakefields in dielectric laser acceleration grating structures, Phys. Rev. Accel. Beams 23, 54402 (2020).

[38] J. Rumble, CRC Handbook of Chemistry and Physics (CRC Press, Boca Raton, FL, 2020), 101st ed.

[39] S. Jalas, M. Kirchen, P. Messner, P. Winkler, L. Hübner, J. Dirkwinkel, M. Schnepp, R. Lehe, and A. R. Maier, Bayesian Optimization of a Laser-Plasma Accelerator, Phys. Rev. Lett. 126, 104801 (2021).

[40] A. Edelen, N. Neveu, M. Frey, Y. Huber, C. Mayes, and A. Adelmann, Machine learning for orders of magnitude speedup in multiobjective optimization of particle accelerator systems, Phys. Rev. Accel. Beams 23, 44601 (2020). 